Service social

\title{
Intervenir auprès des victimes d'intimidation en milieu scolaire : l'empowerment comme approche alternative
}

\section{Marie-Michelle Poulin et Valérie Roy}

Volume 62, numéro 1, 2016

Ensemble contre l'intimidation : une responsabilité partagée

URI : https://id.erudit.org/iderudit/1036333ar

DOI : https://doi.org/10.7202/1036333ar

Aller au sommaire du numéro

Éditeur(s)

École de service social de l’Université Laval

ISSN

1708-1734 (numérique)

Découvrir la revue

Citer cet article

Poulin, M.-M. \& Roy, V. (2016). Intervenir auprès des victimes d'intimidation en milieu scolaire : l'empowerment comme approche alternative. Service social, 62(1), 24-37. https://doi.org/10.7202/1036333ar
Résumé de l'article

Cet article se base sur une recension des écrits dans le cadre d'un projet d'intervention à la maîtrise en service social. Il propose une réflexion critique sur les interventions réalisées auprès de jeunes victimes d'intimidation en milieu scolaire. La recension amène à constater la concentration des objectifs des différents programmes d'intervention autour de la diminution des facteurs de risque d'être victime et de l'acquisition d'habiletés sociales. Malgré la pertinence de ces objectifs, les programmes semblent s'en tenir à une compréhension limitée, et très individuelle, de la problématique. Pour pallier ces limites, l'empowerment, qui permet une analyse des aspects macro-sociaux, est proposée comme approche alternative. Les obstacles possibles à son application en milieu scolaire viennent conclure la réflexion critique. 


\title{
Intervenir auprès des victimes d'intimidation en milieu scolaire : 1'empowerment comme approche alternative
}

POULIN, Marie-Michelle, 1 T.S. Étudiante à la maîtrise en service social École de service social, Université Laval

ROY, Valérie, ${ }^{2}$ Ph.D. T.S. Professeure agrégée École de service social, Université Laval

\section{RÉSUMÉ}

Cet article se base sur une recension des écrits dans le cadre d'un projet d'intervention à la maîtrise en service social. II propose une réflexion critique sur les interventions réalisées auprès de jeunes victimes d'intimidation en milieu scolaire. La recension amène à constater la concentration des objectifs des différents programmes d'intervention autour de la diminution des facteurs de risque d'être victime et de l'acquisition d'habiletés sociales. Malgré la pertinence de ces objectifs, les programmes semblent s'en tenir à une compréhension limitée, et très individuelle, de la problématique. Pour pallier ces limites, l'empowerment, qui permet une analyse des aspects macro-sociaux, est proposée comme approche alternative. Les obstacles possibles à son application en milieu scolaire viennent conclure la réflexion critique.

Mots-clés : Intervention, victime d'intimidation, habiletés sociales, approche alternative, empowerment.

\begin{abstract}
This article is based on a review of the literature that is a part of a master intervention project in social work with groups. It proposes a critical reflection on interventions with young victims of bullying in schools. The review leads to find that most of the objectives of various intervention programs are concentrated around social skills training and the reduction of risk factors of being a bullying victim. Despite the relevance of these goals, the programs seem to stick to a limited understanding, and very personal, issues. To overcome these limitations, empowerment, enabling an analysis of macro-social aspects, is proposed as an alternative approach. Possible resistances to its application in schools close the critical reflection.
\end{abstract}

Keywords: Intervention, victim of bullying, social skills training, alternative approach, empowerment.

\footnotetext{
${ }^{1}$ Marie-Michelle Poulin, T.S., est étudiante à la maîtrise en service social à l'Université Laval. Ses intérêts portent principalement sur l'intimidation en milieu scolaire.

${ }^{2}$ Valérie Roy, Ph.D., T.S., est professeure agrégée à l'École de service social de l'Université Laval. Ses activités de recherche et d'enseignement concernent principalement le service social des groupes et la violence conjugale.
} 


\section{INTRODUCTION}

Au Québec, en juin 2012, le gouvernement a adopté le projet de loi 56 visant à prévenir et à combattre l'intimidation et la violence à l'école. L'intimidation y est définie comme «tout comportement, parole, acte ou geste délibéré ou non à caractère répétitif, exprimé directement ou indirectement, y compris dans le cyberespace, dans un contexte caractérisé par l'inégalité des rapports de force entre les personnes concernées, ayant pour effet d'engendrer des sentiments de détresse et de léser, blesser, opprimer ou ostraciser » (Loi sur l'instruction publique, art. 13, par. 1.1). Bien qu'il soit difficile d'avoir une idée claire de l'ampleur du problème, notamment du fait que les études diffèrent dans la mesure des actes d'intimidation, les données restent préoccupantes. Au Canada, $34 \%$ des garçons et $33 \%$ des filles, âgés de 11 ans, ont été victimes à au moins deux reprises d'actes d'intimidation en 2010 (PHAC, 2014). Au Québec, en ce qui concerne de façon plus spécifique la cyberintimidation, 44,7\% des élèves du secondaire en ont été victimes à quelques reprises en 2010-2011 (Institut de la statistique du Québec, 2013). La situation est encore plus préoccupante pour certains groupes, notamment chez les jeunes en difficulté alors que $30 \%$ d'entre eux seraient victimes d'intimidation de façon régulière (Hong et Espelage, 2012 ; Rose, Espelage et Monda-Amaya, 2009). Ce problème entraîne de nombreuses conséquences pour les jeunes qui en sont victimes (Craig et Pepler, 2003 ; Olweus, 2013 ; Rigby, 2003 ; Takiwaza, Maughan et Arsenault, 2014). Sur le plan psychologique, des problèmes d'anxiété, de dépression et de perte d'estime de soi sont observés, alors que, sur le plan social, l'isolement, l'exclusion et la stigmatisation sont vécus par plusieurs (Rigby, 2003 ; Stassen Berger, 2007). La lutte contre l'intimidation est d'ailleurs devenue une préoccupation majeure pour les milieux scolaires et l'ensemble des communautés (PHAC, 2014) et différentes stratégies de prévention et d'intervention sont proposées.

Faisant suite à la Loi 56, en octobre 2014, le gouvernement du Québec a mis sur pied un forum, diffusé en direct sur le web, afin d'élaborer un plan d'action concerté (ministère de la Famille, 2014a, 2014b). Les acteurs des différents milieux concernés par la problématique ont souligné l'importance de la prévention, de la sensibilisation, de l'éducation et de la formation chez les intervenants-clés. De façon plus spécifique aux jeunes, le développement de l'affirmation de soi et de l'empathie, ainsi que l'acquisition de comportements d'entraide et d'habiletés sociales étaient les principales stratégies proposées (ministère de la Famille, 2014a, 2014b). Certains participants au forum ont toutefois attiré l'attention sur la nécessité d'avoir une compréhension plus large de la problématique et de résister à la tentation de proposer des solutions uniques (ministère de la Famille, 2014a, 2014b). 
S'inscrivant dans ces préoccupations, le présent article propose une réflexion critique sur des interventions menées auprès de jeunes victimes d'intimidation. II repose sur une recension des écrits effectuée dans le cadre d'un essai de maîtrise en service social à l'Université Laval. Les banques de données Child Development \& Adolescent Studies, Social Sciences Full Text, Social Services Abstracts, ERIC, eBook Collection, Education Source, FRANCIS, Psychology and Behavioral Sciences Collection et SocINDEX ont été consultées avec les mots clés suivants: bullying, empowerment, school, school-based, programs, intervention, prevention, best practice, social work with groups. Les mots empowerment, jeunes, interventions, intimidation, école, victimes, service social des groupes ont été utilisés dans les banques de données francophones Cairn et Érudit. Plus d'une centaine de documents ont été consultés et une trentaine de textes centrés sur les interventions ont été approfondis pour appuyer l'analyse critique. Tout d'abord, l'article présente un état des connaissances sur les interventions réalisées auprès des jeunes victimes d'intimidation en milieu scolaire. Par la suite, les limites de ces interventions seront présentées. Finalement, une approche alternative, soit celle de l'empowerment, sera discutée sous l'angle des forces et des obstacles possibles, anticipés sur la base de la recension des écrits, des caractéristiques de l'approche et du milieu scolaire.

\section{DES PROGRAMMES À VISÉE ÉDUCATIVE}

Généralement, les programmes pour lutter contre l'intimidation adoptent un modèle d'intervention scolaire multiniveaux ou school-based programs. Selon le modèle d'intervention scolaire, les school-based programs intègrent des stratégies de prévention à différents paliers, soit la communauté, l'école, la classe, et les acteurs d'une situation d'intimidation (agresseurs/témoins/victimes, parents). Ce caractère inclusif constitue d'ailleurs l'une des forces des school-based programs (Limber, 2011 ; Ttofi et Farrington 2011). Plusieurs d'entre eux découlent de l'Olweus Bullying Prevention Program (OBPP) qui s'avère un bon exemple d'un school-based program (Limber, 2011). Développé en Norvège en 1983, l'OBPP est considéré mondialement comme une référence, notamment puisqu'il est le premier programme à avoir été implanté et évalué à un niveau national (Limber, 2011).

Le volet collectif du OBPP vise la sensibilisation, la conscientisation et l'implication de la communauté, notamment par le soutien matériel ou financier des commerces pour les activités liées au programme (Limber, 2011). L'inclusion de la communauté permet de sensibiliser une plus grande part de la population et d'instaurer un climat de non-violence par la diffusion de stratégies de prévention et d'un message intégratif dans l'ensemble des milieux de vie (Limber, 2011). 
Au niveau de l'école, l'OBPP propose la création d'un comité de coordination de lutte contre l'intimidation. Celui-ci comprend des membres de la direction, du personnel enseignant et non enseignant, des intervenants psychosociaux et parfois des élèves. Pour l'école, le programme prévoit la passation régulière de l'Olweus Bullying Questionnaire (OBQ) afin de colliger les données réelles du phénomène de l'intimidation et de documenter l'évolution de la problématique. De plus, l'OBPP prescrit une formation pour l'ensemble du personnel de l'école, une surveillance accrue de l'établissement et de la cour par les employés, ainsi que l'adoption de règlements clairs quant aux comportements d'intimidation et de violence. Pour l'OBPP, une communication régulière entre les enseignants, les intervenants et les parents est aussi essentielle. L'implication des parents dans des rencontres de classe et lors de situations d'intimidation impliquant leurs enfants, en collaboration avec les enseignants et les intervenants, est recherchée (Ttofi et Farrington, 2011).

Au niveau de la classe, l'OBPP prévoit l'établissement de règlements anti-intimidation, de rencontres éducatives hebdomadaires abordant l'intimidation et d'autres thèmes connexes, ainsi que la dénonciation des événements d'intimidation. Le programme propose aussi à l'ensemble des élèves le renforcement des comportements prosociaux et l'apprentissage d'habiletés sociales, incluant entre autres la capacité d'intervenir dans des situations d'intimidation à titre de pair aidant. L'intégration d'une pédagogie encourageant et valorisant les comportements prosociaux permettrait notamment aux témoins d'agir auprès des victimes afin de les aider ou les soutenir lors de situation d'intimidation (Limber, 2011 ; Ttofi et Farrington, 2011 ; Whitted et Dupper, 2005).

En ce qui a trait aux acteurs impliqués dans une situation d'intimidation, l'OBPP prévoit des interventions individuelles ou des programmes de groupe. Pour les agresseurs, le programme suggère l'application immédiate de conséquences, un suivi auprès du jeune, et un renforcement des comportements non violents. Des rencontres avec les parents de l'agresseur font également partie du programme. Pour les victimes, le programme prévoit des rencontres de soutien, auquel s'ajoute un volet éducatif, misant entre autres sur l'acquisition d'habiletés sociales et de résolution de problèmes. En outre, des rencontres avec les parents sont offertes (Farrington et Ttofi 2009 ; Ttofi et Farrington, 2011 ; Whitted et Dupper, 2005).

Bien qu'ils s'appuient généralement sur l'OBPP, les school-based programs présentent certaines différences, notamment dans les interventions s'adressant aux jeunes victimes. Certains programmes s'en tiennent à une approche psychoéducative centrée sur l'acquisition d'habiletés 
sociales et de résolution de problèmes (Fox et Boulton, 2003a; Whitted et Dupper, 2005), accordant ainsi peu d'espace aux besoins de soutien des jeunes et des parents. Par ailleurs, ces programmes paraissent basés sur la prémisse que des difficultés interpersonnelles et comportementales des jeunes victimes contribuent à leur victimisation (Fox et Boulton, 2003a ; Whitted et Dupper, 2005). Ainsi, il est généralement accepté que l'acquisition d'habiletés sociales favoriserait une meilleure estime de soi, facteur protecteur contre l'intimidation, et réduirait la présence de caractéristiques personnelles augmentant le risque d'être victimisé de nouveau, comme paraitre faible ou effrayé (Fox et Boulton, 2003a; Ttofi et Farrington, 2011 ; Whitted et Dupper, 2005). Dans le même ordre d'idées, plusieurs auteurs affirment que l'acquisition d'habiletés sociales permettrait une modification des comportements de fuite ou d'inaction adoptés par les jeunes victimes lorsqu'ils sont intimidés. De plus, l'intervention ayant pour objectif l'acquisition d'habiletés sociales faciliterait pour les victimes leur inclusion auprès de leurs pairs.

Le Social Skills Training Programme (SST) est un bon exemple d'un programme d'intervention pour les jeunes victimes. Le SST a pour objectif l'acquisition d'habiletés sociales et la réduction des facteurs de risque individuel d'être victimisé (Fox et Boulton, 2003a). De façon plus spécifique, il vise à augmenter les comportements prosociaux, à diminuer les comportements des victimes, dont la soumission, qui les font paraître comme des cibles faciles et à mieux gérer le stress, notamment lors de situations d'intimidation (Fox et Boulton, 2003a, 2003b). Le programme propose huit rencontres pour aborder les thèmes de l'amitié, du langage corporel, de l'affirmation de soi et des stratégies à adopter advenant d'autres situations d'intimidation (Fox et Boulton, 2003b). Des techniques telles que les jeux de rôles, le modeling, les rétroactions et les techniques de relaxation sont utilisées dans l'intervention.

\section{L'efficacité des programmes éducatifs pour les jeunes victimes}

Selon Ttofi et Farrington (2011), la majorité des programmes d'intervention se basent sur l'OBPP, sans toutefois l'appliquer de façon intégrale et sans avoir fait l'objet d'une évaluation systématique. Sur la base d'une méta-analyse de 89 recherches évaluatives (ayant notamment un échantillon minimal de 200 et utilisant des questionnaires auto-rapportés auprès des jeunes), ces auteurs concluent que les school-based programs entraînent une diminution significative de $20 \%$ à $23 \%$ des actes d'intimidation commis. Par contre, la diminution du nombre de victimes, entre $17 \%$ et $20 \%$, ne serait pas significative, possiblement en raison des disparités dans l'application des programmes (fidélité, dosage, durée, formation des enseignants, inclusion de rencontres avec les parents, nombre d'élèves par classe) (Ttofi et Farrington, 2011). Bien que ces 
résultats soient intéressants, il reste qu'un nombre important de victimes continue de subir des préjudices.

En ce qui concerne le programme SST cité précédemment, une recherche évaluative (Fox et Boulton, 2003a) a été réalisée auprès de 15 jeunes victimes ayant participé au programme. En comparaison avec un groupe contrôle $(n=13)$, sur l'ensemble des variables observées, soit la dépression, l'anxiété, l'acceptation par les pairs et l'acceptation sociale, seule l'estime de soi des jeunes ayant pris part au SST s'est améliorée de façon significative. Fox et Boulton (2003a) expliquent ces résultats par les limites du programme à considérer l'interaction entre les émotions et les cognitions qui interfèrent lors des situations d'intimidation, ce qui rendrait d'ailleurs l'application des habiletés sociales et de résolution de problèmes plus difficiles.

\section{Limites des programmes recensés}

Plusieurs autres limites des programmes pour les victimes d'intervention peuvent être soulevées. Afin de cerner ces limites, il s'avère pertinent de revenir à la définition de l'intimidation et de ses composantes. Bien que les définitions varient, les auteurs s'accordent généralement sur quatre critères pour évaluer si une situation constitue de l'intimidation (Olweus, 1994, 1999, 2993, 2013; Stassen Berger, 2007). Le préjudice doit être porté à plusieurs reprises et, deuxièmement, doit être fait de façon intentionnelle. Il doit aussi être posé dans le but de causer des souffrances ou de porter atteinte à l'intégrité physique ou psychique de la personne intimidée. Finalement, l'un des éléments essentiels est la présence d'un déséquilibre de force entre l'intimidateur et sa victime (Olweus, 1994, 1999, 2003, 2013; Stassen Berger, 2007).

Une première limite des programmes recensés a trait à la compréhension de la problématique qu'ils traduisent, soit une vision centrée sur les aspects microsociaux aux dépens des aspects macrosociaux. Bien que les school-based programs soient inclusifs des différents acteurs de la communauté, reconnaissant par le fait même l'importance d'agir sur les facteurs sociaux sousjacents à la problématique, l'intervention auprès des victimes semble occulter totalement ces facteurs sociaux, dans lesquels s'inscrit le déséquilibre de pouvoir entre l'agresseur et sa victime (p. ex. origine ethnique, pauvreté). Ce déséquilibre de pouvoir est sous-tendu par des valeurs de performance, de hiérarchisation et de conformisme prônées au sein de la société (Galitz et Robert, 2014) et qui se répercutent dans les interactions sociales. Les programmes mettent plutôt l'accent sur la diminution des facteurs de risque individuel d'être victime d'intimidation (Farrington et Ttofi, 2009 ; Ttofi et Farrington 2011 ; Whitted et Dupper, 2005). Or, l'intimidation ne tient pas à la seule 
présence de déficits d'habiletés sociales chez les victimes et la réduction aux seuls facteurs de risques individuels peut comporter des effets pervers pour les victimes.

En effet, les objectifs d'intervention d'acquisition d'habiletés sociales, de modification de comportements rendant la victime plus à risque de se faire intimider et la diminution des autres facteurs de risque individuels pourraient envoyer un message blâmant les jeunes intimidés pour l'intimidation vécue. Le concept d'oppression cognitive de Breton (2006) s'avère éclairant à ce sujet. Pour cette auteure, l'oppression cognitive consiste en l'intériorisation, chez les personnes opprimées, des messages véhiculés par les oppresseurs. Ainsi, ces objectifs d'intervention pourraient contribuer à l'oppression cognitive, ce qui amènerait les victimes à s'attribuer le blâme (blame the victim) pour l'oppression vécue, dans ce cas-ci, l'intimidation subie. Les thématiques abordées avec les victimes comme l'amitié et de langage corporel dans le programme SST (Fox et Boulton, 2003a) sont un exemple qui permet d'illustrer le concept d'oppression cognitive. Les victimes, qui selon Olweus (1999) ont possiblement au préalable une image négative d'ellesmêmes, pourraient penser que l'intimidation est due à leurs difficultés d'interagir de façon appropriée avec d'autres jeunes, à leur incapacité de s'intégrer au groupe et à leur langage corporel inadéquat. L'intériorisation de messages véhiculés par ces thèmes, axés sur les déficits sur le plan des habiletés sociales, pourrait s'avérer dommageable chez ces jeunes. Ces messages suggèrent une responsabilisation des victimes, car l'intervention vise la modification de ses comportements, alors que la problématique de l'intimidation est plus complexe et implique un déséquilibre de pouvoir entre l'agresseur et la victime (Olweus, 2013). L'utilisation des techniques comme les jeux de rôle et la relaxation favorise certes l'acquisition d'habiletés sociales (Fox et Boulton, 2003a), mais l'emploi seul de ces techniques pourrait suggérer, selon le concept de l'oppression cognitive de Breton (2006), une responsabilisation de l'expérience d'intimidation. Au contraire, une approche d'empowerment, comme celle proposée par Ninacs (2003) encouragerait plutôt une remise en question et un développement de la conscience critique chez les jeunes victimes quant à la problématique de l'intimidation et à la composante de déséquilibre de pouvoir.

Attribuer aux victimes d'intimidation la responsabilité de la situation dans laquelle elles se trouvent est un phénomène nommé « double victimisation » (Damant, Paquet et Bélanger, 2001). La présence d'une double victimisation et l'intériorisation des propos des agresseurs (« t'es un bon à rien », « t'es niaiseuse ») pourraient augmenter ou aggraver les conséquences vécues par les victimes, dont des comportements d'isolement ou une diminution de la participation sociale. Ces comportements pourraient résulter, par exemple, de l'évitement des autres afin de ne pas se sentir incompétent socialement. II s'agirait là d'un effet paradoxal des programmes qui visent à 
rendre plus compétents les jeunes victimes. Par ailleurs, selon Fox et Boulton (2003a), la décontextualisation de l'intervention psychoéducative rend plus difficile la généralisation des habiletés nouvellement acquises par les jeunes. De plus, les programmes s'adressant aux victimes abordent peu les conséquences de l'intimidation subie. En effet, comme l'évaluation de Fox et Boulton (2003a) l'a démontré, le programme SST ne semble pas avoir eu d'effets sur la dépression, l'anxiété, l'acceptation par les pairs et l'acceptation sociale. Ainsi, en plus de ne pas avoir d'effets sur les conséquences personnelles et sociales, ces programmes pourraient exacerber les conséquences vécues chez les victimes (Graham et Juvonen, 1998).

\section{L'EMPOWERMENT COMME APPROCHE ALTERNATIVE}

En réponse à ces limites, l'empowerment apparaît comme une approche alternative intéressante à mettre de l'avant dans les interventions auprès des jeunes victimes d'intimidation. L'empowerment s'est développée en réaction aux modèles ayant une logique technoscientifique qui attribuent le déficit de pouvoir des individus à des faiblesses personnelles et individuelles (Damant, Paquet et Bélanger, 2001). Ces modèles identifient la source des difficultés des individus dans leurs incapacités individuelles et la sous-utilisation de leurs compétences, dont leurs habiletés sociales. L'intervention vise donc à les responsabiliser, de sorte qu'ils puissent résoudre leurs difficultés par eux-mêmes (Damant, Paquet et Bélanger, 2001). Une intervention s'appuyant sur l'empowerment visera plutôt à proposer un ensemble d'activités afin de permettre aux individus d'augmenter le pouvoir qu'ils détiennent sur leur vie (Swift et Levin, 1987). Mullaly (2010) affirme que le pouvoir ne peut être donné aux individus : eux seuls sont en mesure de le réclamer ou de le reprendre. Pour les jeunes victimes d'intimidation, l'empowerment amènerait à concevoir une intervention qui permettrait aux jeunes de reprendre du pouvoir sur leur vie et de se libérer de l'oppression intériorisée. De plus, l'empowerment est une approche qui met de l'avant les forces et habiletés des individus en perte de pouvoir, notamment par la prise de décision et le développement et la réalisation de projets collectifs. L'empowerment offre aussi une vision d'ensemble de la réalité des individus qui reprennent du pouvoir sur leur vie, entre autres par la réflexion et la mise en commun de leurs expériences avec les membres du groupe, et ce, malgré les conditions difficiles dans lesquelles ils vivent (Damant, Paquet et Bélanger, 2001). Cela étant dit, il existe différents modèles d'intervention s'appuyant sur l'empowerment (Damant, Paquet et Bélanger, 2001) et le modèle proposé par Ninacs (2003) s'avère particulièrement intéressant en fonction des besoins des jeunes victimes d'intimidation. II propose un processus de réappropriation du pouvoir d'agir sur quatre composantes, soit la conscience critique, la participation sociale, les compétences pratiques et l'estime de soi. 
Une intervention s'appuyant sur l'empowerment vise d'abord le développement d'une conscience critique (ou conscientisation) par un processus de réflexion et d'action (Ninacs, 2003). La conscience critique cherche à aider l'individu à réaliser qu'il n'est pas le seul à avoir un problème, que les problèmes individuels et collectifs sont influencés par l'organisation sociale de la société (incluant les structures politiques, sociales, économiques et culturelles) et que la solution de ces problèmes passe par un changement social (Ninacs, 1995). Le développement d'une conscience critique ouvre ainsi la voie à une compréhension plus large de l'intimidation, c'est-à-dire qu'elle résulte d'un déséquilibre de pouvoir (Olweus, 2013), qu'elle est influencée par la façon dont la société est organisée, et qu'elle comporte des causes et des solutions collectives. Par exemple, par un partage de leurs expériences, les jeunes pourront identifier les aspects qui sont communs et qui dépassent leurs caractéristiques individuelles. La discussion cherchera à comprendre comment ces aspects sont plutôt liés aux différences basées sur la classe sociale, l'origine ethnique, le genre, l'apparence physique, ou l'orientation sexuelle, constituant des facteurs de risque de subir de l'intimidation (Olweus, 1999). Ces différences génèrent des rapports sociaux inégaux, caractérisés par la domination de certains groupes qui exercent leur pouvoir, notamment par des remarques dévalorisant les personnes en marge des normes établies (Damant, Paquet et Bélanger, 2001). Ainsi, la prise de conscience collective favorise la réduction de l'autoculpabilisation (Ninacs, 2003).

La conscientisation se réalise notamment par les interactions au sein d'un petit groupe, soit par la participation sociale. La participation des personnes est conçue selon une progression allant de la présence muette aux rencontres à une implication active dans les débats, jusqu'à la prise de décision touchant la vie du groupe, par exemple le choix des sujets ou des activités du groupe (Ninacs, 1995). Plusieurs auteurs s'accordent sur le fait que la participation sociale active est la base de la transformation de son environnement (Breton, 2006; Mulally, 2010). Par leur participation sociale au sein du groupe, les jeunes ayant été victimisés sont ainsi amenés à prendre conscience qu'ils ne sont pas les seuls à vivre ce problème et ils peuvent normaliser leurs expériences, incluant les émotions et les conséquences éprouvées (Ninacs, 1995, 2003). Les interactions au sein du petit groupe permettent aussi aux jeunes de comprendre qu'ils ont une voix et qu'ils peuvent être entendus (Breton, 2006). Ils sont ainsi encouragés à se concevoir comme les principaux acteurs des actions à entreprendre pour reprendre du pouvoir sur leur vie, tout en visant un changement social (Le Bossé, 1996). De plus, considérant que les petits groupes stimulent la création de liens sociaux entre les membres (Ninacs, 1995), il est possible de croire 
que la participation au sein du groupe contribuera à une diminution de l'exclusion sociale, de la solitude et de l'isolement vécu par les jeunes ayant été victimes d'intimidation.

Par ailleurs, la participation sociale au sein du petit groupe a pour effet de favoriser l'acquisition ou la réévaluation des habiletés sociales et des compétences pratiques requises par la participation et l'action (Ninacs, 2003). La prise de parole, le partage de ses idées, le respect du tour de parole des autres membres et de leurs opinions sont quelques exemples des compétences pratiques développées par la participation sociale dans un groupe s'appuyant sur l'empowerment. Ici, le développement des habiletés sociales s'appuie sur les forces des membres et sur leur expérience au sein du groupe, notamment par la conscientisation qui amène un partage entre les membres de leurs connaissances sur l'intimidation. L'acquisition des habiletés sociales se module au gré des interactions avec les membres du groupe. Les interactions positives et respectueuses sont renforcées par les réactions et les rétroactions positives des membres et de l'animateur, alors que les interactions négatives sont recadrées, notamment par l'expression des sentiments face aux actions ou propos blessants par les participants et l'animateur. Ainsi, les objectifs sont centrés sur l'intérêt des membres plutôt qu'uniquement sur la modification de leurs interactions et comportements, ce qui peut permettre des interactions plus spontanées et naturelles entre les membres. Par ailleurs, l'intervention de groupe fait en sorte de recréer le contexte dans lequel s'est produite l'intimidation, soit un groupe de pairs sensiblement du même âge qui interagissent le plus librement possible contrairement, par exemple, à une intervention individuelle ou à des jeux de rôles structurés en groupe. Ce contexte similaire d'interactions libres permet aux jeunes d'expérimenter, à plusieurs reprises et de diverses façons, des façons d'être et d'agir avec leurs pairs. Ces expériences répétées peuvent avoir un effet positif sur le sentiment de compétence lors d'interactions sociales chez les jeunes victimes et, ultimement, favoriser la généralisation des acquis à d'autres situations.

Finalement, la conscience critique, la participation sociale et le développement de compétences par les interactions peuvent contribuer au développement de l'estime de soi. La diminution de l'estime de soi est considérée comme une conséquence importante de l'intimidation (Rigby, 2003 ; Stassen Berger, 2007) et plusieurs auteurs en font un objectif à privilégier dans l'intervention (Fox et Boulton, 2003a ; Ttofi et Farrington, 2011 ; Whitted et Dupper, 2005). Dans une perspective d'empowerment, l'estime de soi se conçoit comme la reconnaissance de ses compétences, par soi-même et par les autres, et la légitimité de son identité propre (Ninacs, 2003). L'amélioration de l'estime de soi permet ainsi de surmonter l'oppression cognitive qui découle entre autres de l'intériorisation des messages véhiculés par les intimidateurs. L'amélioration de 
l'estime de soi dans une perspective d'empowerment favorise le changement et la transformation sociale des rapports sociaux jugés discriminatoires (Damant, Paquet et Bélanger, 2001) plutôt que d'être perçu uniquement en tant qu'impact négatif à diminuer dans une intervention basé sur un modèle psychoéducatif.

En somme, il est possible de penser qu'une intervention basée sur l'empowerment, en plus de permettre la réappropriation du pouvoir sur sa vie, contribuerait à la diminution des conséquences personnelles et sociales chez les victimes, dont l'isolement et la faible estime de soi.

\section{Les obstacles possibles à l'utilisation d'une intervention basée sur l'approche de l'empowerment}

L'intervention issue d'une approche d'empowerment peut s'avérer une alternative aux modèles psychoéducatifs en aidant à mieux comprendre comment le processus de victimisation découle des inégalités sociales. Par contre, il est possible d'envisager certains obstacles quant à son utilisation. L'empowerment est une approche peu abordée dans les écrits scientifiques concernant spécifiquement la problématique de l'intimidation. L'absence de données probantes sur des interventions fondées sur l'empowerment pourrait amener les décideurs à préférer une intervention déjà éprouvée. En outre, l'approche d'empowerment impliquerait un ajustement du rôle des professionnels dans l'intervention. En empowerment, les intervenants doivent partager l'expertise avec les membres, qui détiennent le savoir expérientiel, et adopter un rôle de facilitateur (Le Bossé, 1996). Un tel ajustement pourrait susciter certaines réserves, ou du moins une période d'adaptation. Une approche basée sur l'empowerment requiert aussi du temps et une implication volontaire des participants (Ninacs, 1995). La présence de contraintes structurelles en milieu scolaire pourrait freiner l'implantation de programmes basés sur cette approche. La disponibilité des participants (horaires d'activités scolaires et parascolaires préétablis), la disponibilité des locaux, les diverses demandes à adresser aux parents, à la direction ou au conseil d'établissement pour la réalisation de certaines activités dans le cadre du processus d'empowerment font partie des contraintes avec lesquelles l'animateur et les membres du groupe devraient jongler dans la réalisation de l'intervention. Finalement, même si le contexte d'intervention de groupe dans lequel s'inscrit l'empowerment peut favoriser la généralisation des acquis, il resterait à évaluer dans quelle mesure ces acquis peuvent se traduire dans d'autres groupes sociaux fréquentés par ces mêmes jeunes (Ninacs, 1995). En effet, il serait intéressant de voir si les acquis sur le plan de la conscience critique, de la participation sociale, des 
compétences pratiques peuvent s'observer dans d'autres contextes comme à la Maison des jeunes du quartier ou au camp de jour pour ne donner que deux exemples.

\section{Conclusion}

Cet article a proposé une réflexion critique sur les interventions offertes aux jeunes victimes d'intimidation. La majorité des interventions recensées sont fondées sur la diminution du risque d'être victimes par l'acquisition d'habiletés sociales plutôt que sur les composantes de déséquilibre de pouvoir et sur les conséquences de la victimisation. Le concept de l'oppression cognitive de Breton (2006) suggère que les interventions visant la diminution des risques et l'acquisition d'habiletés sociales pourraient aggraver les conséquences de l'intimidation par la responsabilisation des victimes et par l'intériorisation des messages opprimants véhiculés à leur endroit. Dans le but de pallier ces limites, l'article a proposé une approche d'empowerment pour guider les programmes qui, en plus de s'adresser au déséquilibre de pouvoir caractérisant la problématique d'intimidation, offre un processus permettant la reprise du pouvoir sur sa vie et la diminution des conséquences telles que l'isolement et la perte d'estime de soi. Bien que les effets d'une telle approche restent à évaluer, incluant le transfert des acquis dans d'autres contextes sociaux, cette approche, par ses bénéfices possibles et sa cohérence avec la définition du problème, nous semble intéressante à considérer dans le cadre de l'élaboration du plan d'action concerté du Gouvernement du Québec. Dans ce contexte, il serait judicieux de diversifier les actions entreprises pour venir en aide aux jeunes victimes d'intimidation afin qu'elles ne soient pas uniquement centrées sur les individus et leurs facteurs de risque personnels, mais qu'elles offrent plutôt un espace de conscientisation critique et des possibilités de reprises de pouvoir sur sa vie.

POULIN, Marie-Michelle, T.S. Étudiante à la maîtrise en service social École de service social, Université Laval

ROY, Valérie, Ph.D. T.S. Professeure agrégée École de service social, Université Laval 


\section{RÉFÉRENCES}

Breton, M. (2006). «An Empowerment Perspective », dans C.D. Garvin, L.M. Gutiérrez, L.M. et M.J. Galinsky (dir.), Handbook of Social Work with Groups, New York, Guilford Press, p. 5875.

Craig, W.M., et D.J. Pepler (2003). « Identifying and Targeting Risk for Involvement in Bullying and Victimization », Canadian Journal of Psychiatry, vol. 48, n 9, p. 577-582.

Damant, D., J. Paquet et J. Bélanger (2001). « Recension critique des écrits sur l'empowerment ou quand l'expérience de femmes victimes de violence conjugale fertilise des constructions conceptuelles », Recherches féministes, vol. 14, n² 2, p. 133-154.

Farrington, D.P., et M.M. Ttofi (2009). « Reducing School Bullying: Evidence-Based Implications for Policy », Crime and Justice, vol. 38, n 1, p. 281-346.

Fox, C.L., et M.J. Boulton (2003a). « A Social Skills Training (SST) Programme for Victims of Bullying », Pastoral Care in Education, vol. 21, n² 2, p. 19-26.

Fox, C.L., et M.J. Boulton (2003b). « Evaluating the effectiveness of a social skills training (SST) programme for victims of bullying », Educational Research, vol. 45, n 3, p. 231-247.

Galitz, T., et D. Robert (2014). «Governing bullying through the new public health model: a Foucaultian analysis of a school anti-bullying programme », Critical Public Health, vol. 24, $\mathrm{n}^{\circ} 2$, p. 182-195.

Graham, S., et J. Juvonen (1998). « Self-Blame and Peer Victimization in Middle School: An Attributional Analysis », Developmental Psychology, vol. 34, nº 3, p. 587-599.

Hong, J.S., et D.L. Espelage (2012). «A review of research on bullying and peer victimization in school: An ecological system analysis », Aggression and Violent Behavior, vol. 17, $n^{\circ}$ 4, p. 311322.

Institut de la Statistique du Québec (2013). L'Enquête québécoise sur la santé des jeunes du secondaire 2010-2011, tome 2 : Le visage des jeunes d'aujourd'hui : leur santé mentale et leur adaptation sociale, Québec, Gouvernement du Québec. Accessible en ligne : $<$ http://www.stat.gouv.qc.ca/statistiques/sante/enfants-ados/adaptation-sociale/sante-jeunessecondaire2.pdf>.

Le Bossé, Y. (1996). «Empowerment et pratiques sociales : illustration du potentiel d'une utopie prise au sérieux », Nouvelles pratiques sociales, vol. 9, nº 1, p. 127-145.

Limber, S. P. (2011). Development, Evaluation, and Future Directions of the Olweus Bullying Prevention Program. Journal of School Violence, 10(1), 71-87.

Ministère de la Famille. (2014a). Forum sur la lutte contre l'intimidation : Synthèse. Accessible en ligne : <http://www.mfa.gouv.qc.ca/fr/publication/Documents/synthese-forum.pdf>.

Ministère de la Famille. (2014b). Forum sur la lutte contre l'intimidation. Communication présentée à Québec, vidéodiffusée en direct. 
Mullaly, B. (2010). Challenging Oppression and Confronting Privilege: A Critical Social Work Approach ( $2^{\mathrm{e}}$ éd.), Don Mills (ON), Oxford University Press

Ninacs, W.A. (1995). «Empowerment et service social : approches et enjeux », Service social, vol. $44, n^{\circ} 1$, p. 69-93.

Ninacs, W.A. (2003). L'empowerment et l'intervention sociale, Montréal, Centre de documentation sur l'éducation des adultes et la condition féminine. Accessible en ligne: <http://bv.cdeacf.ca/EA_PDF/1159.pdf>.

Olweus, D. (1994). « Bullying at School: Basic Facts and Effects of a School Based Intervention Program », Journal of Child Psychology and Psychiatry, vol. 35, nº 7, p. 1171-1190.

Olweus, D. (1999). Violence entre élèves, harcèlements et brutalités. Les faits, les solutions, Paris, ESF éditeur.

Olweus, D. (2003). « A Profile of Bullying at School », Educational Leadership, vol. 60, n 6, p. 1217.

Olweus, D. (2013). "School bullying: development and some important challenges », Annual Review of Clinical Psychology, vol. 9, p. 751-780.

PHAC (Public Health Agency of Canada). (2014). Health Behaviour in School-Aged Children. Trends Report 1990-2010. Ottawa, Public Health Agency of Canada. Accessible en ligne : $<$ http://www.phac-aspc.gc.ca/hp-ps/dca-dea/prog-ini/school-scolaire/behaviourcomportements/assets/pdf/trends-tendances-eng.pdf $\geq$.

Rigby, K. (2003). «Consequences of Bullying in Schools », Canadian Journal of Psychiatry, vol. $48, n^{\circ} 9$, p. 583-590.

Rose, C.A., D.L. Espelage et L.E. Monda-Amaya (2009). « Bullying and victimisation rates among students in general and special education: A comparative analysis », Educational Psychology, vol. 29, n 7, p. 761-776.

Stassen Berger, K. (2007). « Update on bullying at school: Science forgotten? », Developmental Review, vol. 27, n 1, p. 90-126.

Swift, C., et G. Levin (1987). « Empowerment : An emerging mental health technology », Journal of Primary Prevention, vol. 8, $\mathrm{n}^{\text {os }} 1-2$, p. 71-93.

Takizawa, R., B. Maughan et L. Arsenault (2014). « Adult Health Outcomes of Childhood Bullying Victimization: Evidence From a Five-Decade Longitudinal British Birth Cohort », The American Journal of Psychiatry, vol. 171, nº 7, p. 777-784.

Ttofi, M.M., et D.P. Farrington (2011). «Effectiveness of school-based programs to reduce bullying: a systematic and meta-analytic review », Journal of Experimental Criminology, vol. 7, $\mathrm{n}^{\circ} 1$, p. 27-56.

Whitted, K.S., et D.R. Dupper (2005). « Best Practices for Preventing or Reducing Bullying in Schools », Children and Schools, vol. 27, nº 3, p. 167-175. 